\title{
Transnationalität und soziale Ungleichheit
}

\author{
Jana Schäfer
}

\begin{abstract}
Grenzüberschreitende soziale Praxis, dauerhafter Waren- und Wissensverkehr und soziale Beziehungsgeflechte jenseits von Nationalstaaten bedingen sich gegenseitig. Die Migrationsforschung beschreibt diese grenzüberschreitenden Beziehungsnetze mit dem Begriff Transnationalität. Dieser Beitrag skizziert relevante theoretische und methodologische Perspektiven und ihre Leerstellen. Darüber hinaus stellt er die Fruchtbarkeit der sozialräumlichen Perspektive für die Grenzforschung heraus und diskutiert die Intersektionen von Transnationalität mit Klasse, Geschlecht und race/Ethnizität.
\end{abstract}

\section{Schlagwörter}

Transnationalität, Migration, Klasse, Geschlecht, race/Ethnizität

\section{Einleitung: von Migrant*innen zu Transmigrant*innen}

Die Grenzforschung rückt Grenzen und Grenzziehungen ins Zentrum empirischer und theoretischer Überlegungen. Sie arbeitet derzeit mit einem erweiterten Verständnis von Grenzen, das Theorien über politisch-geografische und soziokulturelle Grenzziehungen miteinander verknüpft (vgl. Haselsberger 2014; Bossong et al. 2017; Gerst et al. 2018). Grenzen werden dadurch als komplexe und dynamische Prozesse fokussiert, die materielle und immaterielle Dimensionen bündeln, um soziale Gruppen und Ordnungen voneinander getrennt zu konstruieren (vgl. ebd.). Es handelt sich bei Grenz(ziehung)en also um Prozesse der Aushandlung von sozialen Ordnungen, die auf räumliche, temporale und sachliche Dimensionen hinweisen. Dieser Beitrag fokussiert ergänzend dazu und in Auseinandersetzung damit aus soziologischer Perspektive grenzüberschreitende soziale Beziehungen.

Migrant*innen und (Im-)Mobile organisieren ihr Leben in der Regel in einem Spannungsverhältnis zwischen politisch-territorialer Grenzpraxis (z.B. Citizenship und Passzugehörigkeit) und sozialen Beziehungen auf Distanz. Die Transnationalitätsforschung stellt mit dem Analysefokus auf grenzüberschreitende soziale Beziehungen beispielhaft die Analyseeinheit von nationalstaatlich orientierten Gesellschaftstheorien, nämlich die Kongruenz von Nation, Staat, Kultur und Gesellschaft (Containerstaat), infrage (vgl. Wimmer/Glick Schiller 2003). Beispielsweise kritisierte Andreas Wimmer in den 1990er-Jahren, dass sich Migrationsforscher*innen primär mit der Integration von Migrant*innen in Ankunftsstaaten beschäftigten. Transnationalitätsforschung ist eine Reaktion auf diese Kritik am methodologischen Nationalismus (vgl. Amelina/Faist 2012).

Die Terminologie Transnationalität ${ }^{1}$ entstammt der interdisziplinären Migrationsforschung. Die Sozialanthropologinnen Linda Basch, Nina Glick Schiller und Cristina Szanton-Blanc (1994) führten die Begriffe transnationale Migration und Transmigrant*in ein, um Migration nicht mehr im Stile der Integrationsforschung als unilinearen, einmaligen und problemverursa-

1 Gebräuchlich ist auch der Begriff Transnationalismus, der auch als Ismus im Sinne einer politischen Ideologie missverstanden werden kann (vgl. Faist et al. 2014). Transnationalität weist im Gegenzug darauf hin, dass es sich um ein (messbares) Merkmal einer Person, eines Netzwerks oder einer Praxis handelt. 
chenden Prozess beschreiben zu müssen. Der Begriff Transnationalität bezeichnet demnach dauerhafte grenzüberschreitende Praxis von Migrant*innen zwischen zwei oder mehreren Ländern, um die sich netzwerkbasierte soziale Räume bilden. Diese transnationalen Netzwerke versorgen Migrant*innen mit lebenswichtigen Ressourcen (vgl. Basch et al. 1994; Haas 2010). Grenzüberschreitend meint hier demzufolge, dass Migrant*innen die territorial-politische Grenz- und Zugehörigkeitspraxis von Nationalstaaten überwinden bzw. simultan an multiplen Kontexten partizipieren.

Die Fokusverschiebung auf Transnationalität ermöglicht es der Migrations- und Mobilitätsforschung, dem Gebot der Reflexivität gerecht zu werden, also die Bedingungen eines Phänomens relativ vollständig abzubilden (vgl. Resch 2014). Die Grenz- und Migrationsforschung muss demnach die Komplexität der Handlungsbedingungen und Machtgefälle zwischen Migrant*innen und Immobilen einfangen und das Zusammenwirken von individuellen Migrationsentscheidungen und (supra)nationalen Migrations- und Grenzregimen analysieren. Durch diese Fokusverschiebung verschwimmt darüber hinaus die Differenz zwischen Migrant*innen und Bürger"innen. Auch Nichtmigrant*innen sind in transnationale Netzwerke involviert: z.B. durch Internet und Telekommunikation (vgl. Büscher/Urry 2009) oder transnationale kapitalistische Wirtschaft (vgl. Sassen 2001). Folglich ist jede* $\mathrm{r}$ zu einem bestimmten Grad transnational. Die Transnationalitätsforschung liefert für die Grenz- und Migrationsforschung also eine wichtige Ergänzung, weil sie die Alltagspraxis diesseits, jenseits und mit der Grenze beleuchtet.

Dieser Beitrag diskutiert zunächst die theoretischen und empirischen Potenziale von Transnationalitätstheorien (Abschnitt 2). Anschließend wird die intersektionelle Interferenz von Transnationalität mit Klasse, Geschlecht und race diskutiert. Damit soll gezeigt werden, dass (sozial)räumliche Distanz und Nähe in der Praxis in ungleiche Lebenschancen übersetzt wird (Abschnitt 3). Schließlich weist dieser Beitrag auf einige Leerstellen und Potenziale innerhalb der jüngsten disziplinären Debatten hin (Abschnitt 4).

\section{Transnationalität: Sozialräumlichkeit empirisch erforschen}

Transnationalitätstheorien betrachten die Sozialräumlichkeit von Alltags- und institutionellen Praktiken, die durch geografische Distanz und nationalstaatliche Institutionen gerahmt sind. Transnationalitätsforschung sieht sich demnach grenzüberschreitende soziale Beziehungen im Kontext von, aber nicht beschränkt auf Nationalstaaten an. Dieser Abschnitt stellt exemplarisch Theorien der Transnationalitätsforschung vor. Er diskutiert zum einen die unterschiedlichen Analyseeinheiten der Transnationalitätstheorien und fragt zum anderen, welche epistemologischen und methodologischen Überlegungen über die Kritik des methodologischen Nationalismus hinaus reflexivitätsfördernd sind (vgl. Amelina/Faist 2012).

\subsection{Die Analyseeinheit(en) der Transnationalitätstheorien}

Transnationalität bezeichnet die grenzüberschreitende soziale Praxis von Migrant*innen zwischen zwei oder mehreren Ländern. Als Konsequenz grenzüberschreitender Praxis entstehen transnationale soziale Beziehungen, die Migrant"innen beispielsweise Zugang zu Wissensnetzwerken gewähren und Krisensituationen auffangen (vgl. Haas 2010). Diese Praxis nimmt 
vielfältige Formen an: familiäre, freundschaftliche, religiöse, ökonomische, organisatorische oder politische Beziehungen. Innerhalb dieser bilden Migrant*innen ihre Identität, treffen Entscheidungen und übernehmen reziprok Verantwortung (vgl. Basch et al. 1994). Zu den relevanten empirischen Dimensionen gehören (sachliche) Sphären und Felder (ökonomisch, politisch etc.), Akteur*innen (kollektive, individuelle und Organisationen), die Komposition der Verbindungen (ihre Reichweite, Dauerhaftigkeit etc.), entstehende soziale Formationen und die Inhalte der Transaktionen (vgl. Boccagni 2012, S. 296).

Während sich alle Transnationalitätstheorien mit grenzüberschreitender Praxis beschäftigen, gewichten sie die Analyseeinheiten unterschiedlich. Peggy Levitt und Nina Glick Schiller (2004) betrachten grenzüberschreitende Netzwerkstrukturen als transnationale soziale Felder. Sie vereinen damit Bourdieus Feldtheorie (1996) mit dem Konzept der Multilokalität bzw. Simultanität, das besagt, dass Migrant*innen dauerhaft institutionelle Strukturen in multiplen Orten nutzen (vgl. Levitt/Glick Schiller 2004). Sie untersuchen, wie Ressourcen verteilt werden und Machtasymmetrien die Lebenschancen der Akteur*innen bestimmen. Thomas Faists (2000) Theorie Transnationaler Sozialräume (TSR) wiederum beschäftigt sich mit den unterschiedlichen Formen transnationaler Beziehungen (ties). Mit unterschiedlichen Graden an Formalisierung und Dauerhaftigkeit gehen Formen von Reziprozität und Solidarität einher, die Zugang zu Gütern, Dienstleistungen, Wissen, Rechten etc. ermöglichen. Faist differenziert damit zwischen stabilen und flüchtigen Formen grenzüberschreitender Beziehungen und fokussiert stärker auf Typen, wie z.B. Familiennetzwerke, Diasporas, Organisationen oder soziale Bewegungen.

Ludger Pries (2008) kritisiert in diesem Zusammenhang essenzialistische Raumkonzepte: TSR sind deterritorialisierte polizentrische (Macht-)Räume um soziale Beziehungen von individuellen und kollektiven Akteurinnen. Diese Perspektive betont zum einen die Simultanität der Lebenskontexte, zum anderen stellt sie auch die Machtdifferenzen zwischen Mobilen und Immobilen heraus. Mit dem Vorschlag eines methodologischen Kosmopolitanismus schließlich stellen Ulrich Beck und Nathan Sznaider (2006) zusammenfassend die Frage, welche Analyseeinheiten und Methoden soziales Handeln im Rahmen globaler ökologischer, ökonomischer und politscher Auseinandersetzungen rekonstruieren können. Sie argumentieren, dass Phänomene wie Klimawandel, Terrorismus oder globale Wirtschaft sowohl die territorialen als auch die Gemeinschaftsaspekte des Nationalstaats überwinden. So muss Handlung ebenfalls jenseits nationalstaatlicher Parameter erklärt werden. Diese Diskussion der Transnationalitätsforschung, die auch für die Grenzforschung relevant ist, stellt hier eine ganz zentrale Frage. TSR existieren jenseits von und im Spannungsverhältnis zu Grenzen: Wie also untersucht man dieses Verhältnis?

\subsection{Epistemologische und methodologische Konsequenzen einer transnationalen Perspektive}

Transnationalitätstheorien entdecken grenzüberschreitende soziale Beziehungen als historisch relativ neue Phänomene, die im Rahmen historisch-kontingenter, weltumspannender „entangled histories“ (vgl. Randeria 2002) auftreten. Die Debatte um Eurozentrismus in der Migrations- und Grenzforschung, die unter den Stichworten „multiple modernities“ (vgl. Eisenstadt 2000) und „provincializing Europe“ (vgl. Chakrabarty 2000) geführt wurde, stellt den zeitli- 
chen und räumlich-territorialen Kontext von Analyseeinheiten noch stärker infrage. Die Kritik lautet, dass Analyseeinheiten wie Nation, Geschlecht oder race von den westlichen Sozialwissenschaften als Herrschaftsinstrumente erfunden wurden und weiter als solche Verwendung finden. Sie betont aber statt der Analyseebene, wie Beck und Sznaider (2006) vorschlagen, eher die historische Verwobenheit und Gleichzeitigkeit von Sozialräumlichkeiten. Diese Kritik sagt uns, dass zur Analyse von transnationalen Netzwerken die Untersuchung der benötigten Infrastruktur gehört. Beispielsweise ist demnach die Ausbeutung eines transnationalen, rassifizierten und gegenderten Prekariats in Produktions- und Reproduktionsarbeit über geografisch verstreute Räume hinweg als Kontextbedingung für die Mobilitätsstrategien von (weißen) Individuen im Westen zu betrachten (vgl. Sassen 2001; s. Abschnitt 3.2).

Im Ergebnis beschäftigt sich Transnationalitätsforschung also mit historisch gerahmten sozialen Netzwerken, ihrer Einbettung in institutionelle Strukturen und der machtsensiblen Verhandlungspraxis von Akteur*innen. Für die empirische Forschung bedeutet dies u.a. die ständige Reflexion der eigenen Positionalität im Forschungsprozess und bei der Theorieverwendung (vgl. Amelina/Faist 2012). Methoden und Methodologien müssen demnach Machtasymmetrien reflektieren und sehen dafür beispielsweise partizipative Forschung (vgl. Mato 2000), simultane Forschung in mehreren Kontexten (multi-sited ethnography; vgl. Marcus 1995) oder mobile methods (vgl. Büscher/Urry 2009) vor. Im Rahmen des mobility turns wurde darüber hinaus das Verhältnis von Stasis und Mobilität in territorialer und zeitlicher Dimension durch Begriffe wie flow neu gerahmt. Diese Autor"innen nehmen wiederum von der sozialen Praxis als solcher Abstand und fokussieren stärker strukturelle Aspekte (vgl. Glick Schiller/Salazar 2013; vgl. dazu auch Nail in diesem Band).

Das Verschieben der Analyseeinheiten lässt sich als ein Ergebnis der Auseinandersetzung der Kultur- und Sozialwissenschaften mit Imperialismus, Nationalismus und Rassismus und deren Folgen verstehen (vgl. Randeria 2002; Mato 2000). Weder die Analyseeinheit oder Analyseebene (vgl. Brenner 1998) noch der zeitliche oder territoriale Rahmen sind letztlich für die Untersuchung von (im)mobilen Personen ,natürlich` vorgegeben. Vielmehr sind sie im Rahmen empirischer Forschung zu entdecken. Grenz(ziehung)en sind wiederum als politisch-territoriale und soziale Dimension bei der Untersuchung transnationaler Mobilität zentral, da sie die grenzüberschreitende Praxis von Individuen und Kollektiven rahmen (vgl. Schindler in diesem Band). Bevor Abschnitt 4 auf neuere Entwicklungen hinweist, diskutiert Abschnitt 3 zunächst Transnationalität als Achse sozialer Ungleichheit, die mit anderen Achsen der Ungleichheit interferiert.

\section{Transnationalität als Ungleichheitsachse}

Transnationale Praxis kann den Verlauf einer Migrant*innenbiografie stark beeinflussen. Wenn man soziale Beziehungen über geografische Distanz und politische Grenzen hinweg als Merkmal versteht, dann kommt Transnationalität als eine Achse der Ungleichheit in den Blick. Ein stabiles transnationales Netzwerk ermöglicht Personen einen sicheren, vorübergehenden oder dauerhaften Ortswechsel oder den Austausch von Waren und Wissen (vgl. Faist et al. 2014). Sozialräumliche und temporale Distanz und Nähe können beispielsweise zur Exklusion von Rechten eingesetzt werden. Die Dichte an grenzüberschreitenden Beziehungen und darauf aufbauenden Handlungsmöglichkeiten kann damit ebenso als Achse der Ungleichheit analysiert werden. Zugang zu Ressourcen wird jedoch nicht allein über Transnationalität geregelt, 
sondern in der Interferenz mit anderen Formen sozialer Privilegierung und Benachteiligung wie Klasse, Geschlecht und race/Ethnizität.

Die Idee der Interferenz unterschiedlicher „systems of oppression“ (Collins 1990, S. 18) wurde innerhalb der Critical Race und Feminist Theory der USA formuliert. Strömungen dieser Kritik finden sich auch in Deutschland (vgl. z.B. Oguntoye et al. 1986). Schwarze, migrantische oder jüdische Frauen fanden und finden ihre Lebenslage in der Kritik weißer Feminist*innen, Marxist*innen oder schwarzer Bürgerrechtler*innen nicht berücksichtigt. Die Afroamerikanische Juristin Kimberle Crenshaw (1989) formulierte die Interferenz von verschiedenen sozialen Benachteiligungen in der Terminologie Intersektionalität ${ }^{2}$. Intersektionalität hebt dabei die Heterogenität innerhalb der im kategorialen Denken als geschlossen gedachten Gruppen hervor. Crenshaw zeigt diese Heterogenität in den untersuchten Fällen von Einstellungsdiskriminierung bei General Motors daran, wie schwarze Frauen dem geltenden Recht nach weder mit den schwarzen Männern noch mit den weißen Frauen eine gemeinsame (rechtsfähige) Gruppe bildeten (vgl. ebd.).

Im Weiteren werden die Ergebnisse der Forschung um die Interferenzen von Transnationalität mit Klasse, Geschlecht und race angerissen. Diese drei ursprünglichen Kategorien der Intersektionalitätsforschung wurden mittlerweile um weitere Kategorien wie Alter, Sexualität, Gesundheit bzw. dis/ability und Raum ergänzt (vgl. Lutz/Amelina 2017). Nationalgesellschaftlich orientierte Theorien übersehen, dass Klasse, Gender und race immer auch quer zu Nationalstaaten existieren. Wir betrachten Achsen der Ungleichheit hier als Demarkationslinien, also als Grenzziehungen (vgl. Bossong et al. 2017), um die herum Privilegierungen und Benachteiligungen (dauerhaft) ausgehandelt werden. Politisch-territoriale Grenzen werden dabei vor allem in ihrer rechtlichen Dimension thematisiert. Die Diskussion ihrer Interferenzen mit Transnationalität beleuchtet die soziale Einbettung der räumlichen Aspekte von sozialer Ungleichheit.

\subsection{Transnationalität und Klasse}

Theorien der Transnationalen kapitalistischen Klasse (TCC) verschieben die Kategorie Klasse auf eine transnationale Analyseebene, um Funktionseliten einer globalen Gesellschaft zu konzipieren. Nach Leslie Sklair (2001) besteht die TCC aus Eliten in den Bereichen Wirtschaft, Politik, Medien und Technik, die zum Teil in Konkurrenz zu national organisierten Eliten stehen und mit einer gemeinsamen, proglobalkapitalistischen Weltanschauung ausgestattet sind. William I. Robinson (2004) postuliert darüber hinaus ein gemeinsames Klassenbewusstsein und die Deterritorialisierung von globalen kapitalistischen Wirtschaftsbeziehungen. Transnational meint hier ein freischwebendes, von nationalen Zwängen gelöstes soziales Netzwerk, wofür typischerweise der Weltwirtschaftsgipfel in Davos angeführt wird. Sowohl Sklair als auch Robinson sparen Sozialräumlichkeit und institutionelle Grenzen, ob nun lokal, national oder transnational, aus ihrer Theorie aus, was eine Leerstelle der TCC-Theorien darstellt (vgl. Carroll 2014). Diese Forschung könnte von der Einbettung in die Transnationalitäts- und Grenzforschung profitieren.

2 Zum Verständnis der Kritik und Weiterentwicklung des Begriffs vgl. Lutz/Herrera Vivar/Supik (2011) und Lutz/ Amelina (2017). 
Die dazugehörige Kehrseite, das transnationale Prekariat, umfasst diejenigen, die über die Mechanismen des globalen Kapitalismus ausgebeutet werden. Migrant*innen sind exemplarisch dafür anzusehen, wie der Antagonismus zwischen Kapital und Arbeit weltweit zu einer Prekarisierung von Arbeitsverhältnissen führt (vgl. Standing 2011). Das transnationale Prekariat sei seinerseits ob fehlender Organisation nicht wirkfähig, aber strukturell vorhanden (vgl. Munck 2013) ${ }^{3}$. Ein weiterer blinder Fleck der TCC-Theorien, nämlich postkoloniale Beziehungen, führt dazu, dass sie auf die Reprekarisierung von kapitalistischen Beziehungen im Westen fokussieren, die durch das Zurückfahren des territorialen Wohlfahrtstaats entstehen. Für (un)dokumentierte Migrant*innen im Westen und für die Mehrheit der Welt (Globaler Süden) sind prekäre Arbeitsverhältnisse keineswegs neu (vgl. ebd.; siehe auch Mezzadra/Neilson in diesem Band).

Man kann Klasse über den Klassenantagonismus hinaus als soziales Netzwerk begreifen. Mit Transnationalität interferiert Klasse dergestalt, dass Transnationalität einerseits zu stärkeren und machtvollen sozialen Beziehungen jenseits nationaler Zugehörigkeit führt (TCC). Andererseits gehören Individuen einer zusätzlich freigesetzten, von den (bedingten) Sicherheiten nationaler Netzwerkkontexte ausgeschlossenen Gruppe an (z.B. transnationale Organisation von Sorgearbeit, Abschnitt 3.2).

\subsection{Transnationalität - Geschlecht - Sexualität}

Transnationalität kann Möglichkeitsräume für marginalisierte Individuen und Gruppen eröffnen. Transnationale Mobilität ist jedoch immer gegendert und sexualisiert. Die Diskurse und (nationalstaatlichen) Regulierungen von Migration setzen Geschlecht und Sexualität als zentrale Ankerpunkte, wenn sie beispielsweise Familiennachzug über Heteronormativität regulieren oder Zugang zu Arbeitsmärkten nach Geschlecht segregieren. Darüber hinaus wurde lange Zeit in der Migrationsforschung übersehen, dass etwa die Hälfte der Migrant*innen weltweit weiblich ist (vgl. Castles et al. 2014). Nicht zuletzt deshalb bietet die Interferenz von Transnationalität, Geschlecht und Sexualität, die hier nur beispielhaft angerissen wird, viele Anknüpfungspunkte für zukünftige Forschung (siehe dazu Bruns in diesem Band).

Care oder Sorgearbeit ist einer der wichtigsten Arbeitsmärkte für Migrant"innen weltweit. ${ }^{4}$ Gemeint sind damit Kinderbetreuung, Hausarbeit und die Pflege von Alten und Kranken, die einem traditionellen Rollenbild entsprechend als unbezahlte Arbeit an Frauen verantwortet wird (vgl. Lutz/Amelina 2017). In der Nachkriegsperiode realisierte sich zunächst das Alleinverdiener-Hausfrauen-Modell für einen Teil der westlichen Länder, aber sowohl in Kapitalismus als auch Kommunismus wurde langfristig die Mitarbeit von Frauen auf dem Arbeitsmarkt benötigt. Wie bespielweise Anna Triandafyllidou und Sabrina Marchetti (2015) zeigen, führte die zunehmende Beteiligung von Frauen in der Erwerbsarbeit zur Nachfrage nach (schlecht bezahlter) Sorgearbeit, die von Migrant*innen übernommen wurde. Frauen im Globalen Norden können von diesen Verhältnissen profitieren (mehr frei verfügbares Einkommen unabhängig von Familieneinkommen), Gender-Pay-Gap und prekäre Arbeit verhindern jedoch häufig, dass

3 Gleichzeitig ist aber auch zu bedenken, dass Klasse in einem Kontext die Klassenzugehörigkeit in einem anderen beeinflussen kann (vgl. Kelly 2012).

4 Die ILO schätzt, dass 11,5 Millionen Migrant*innen weltweit in Haushalten beschäftigt sind, davon ca. $75 \%$ Frauen (vgl. ILO 2015). 
sie die Sorgearbeit in Anspruch nehmen. Die Anwerbung von Migrant*innen entlastet Wohlfahrtsstaaten und belastet gleichzeitig die Familienbeziehungen der Migrant*innen.

Rhacel Parreñas (2005) sowie Deborah Bryceson und Ulla Vuorela (2002) zeigen, dass CareMigration dazu führt, dass Familie auch in Auseinandersetzung mit nationalstaatlichen und globalen Ordnungen stattfindet. Biologische und soziale Familienmitglieder sind aufgrund multipler moralischer und rechtlicher Normen dazu motiviert, Verantwortung für die Familie zu teilen. Transnationale Familien sind jedoch zusätzlich gefordert, da sie über räumliche Distanz Bindungen erzeugen müssen (vgl. Baldassar/Merla 2014). Sie haben multiple rechtliche und kulturelle Kontexte als Bezugsgrößen, die ihre Familienpraxis beeinflussen, etwa indem Familienzusammenführung oder das Übertragen von Rechten auf Angehörige verhindert wird (vgl. Barglowski et al. 2015) oder besondere Betonung auf die Verantwortung von Familien für beispielsweise die nationale Wirtschaft gelegt wird (vgl. Shinozaki 2015). Dies kann dazu führen, dass Mitglieder derselben Familie unterschiedliche Rechte und Zugänge zu Ressourcen haben, und damit u.a. gegenderte Machtungleichheiten verursachen (vgl. Parreñas 2005).

Im Kontext von Care-Migration ist die Abwesenheit von transnationalen Müttern ein häufiges Phänomen. Ihre Abwesenheit kann akzeptiert sein (vgl. Olwig 2014) oder als Versagen interpretiert werden (vgl. Lutz/Palenga-Möllenbeck 2010). Die Abwesenheit von Vätern ist im klassischen Modell der Nuklearfamilie normalisiert, z.B. bei Berufen wie Seefahrer oder Außendienstmitarbeiter. Moderne Kommunikationstechnologien wie Videochats können Präsenz im Leben ihrer Familie vereinfachen (vgl. Madianou/Miller 2011), ersetzen jedoch - wie Helma Lutz und Eva Palenga-Möllenbeck (2010) anmerken - körperliche Nähe und spontane Zusammenkunft nicht. Lutz (2018) konstatiert darüber hinaus, dass Sorge- und Hausarbeit innerhalb der Familien nicht umverteilt wird, weil Väter für Sorgearbeit wenig Anerkennung bekommen. Mutterschaft als soziale Beziehung in transnationalen Familien ist demnach von besonderen Herausforderungen betroffen, die sowohl von der Ungleichheitsachse Geschlecht als auch von der Ungleichheitsachse Transnationalität (re)produziert werden.

Eine weitere Komponente in transnationalen sozialen Beziehungen ist das Praktizieren und Regulieren von Sexualität. Staatliche Biopolitik fokussiert stark auf Sexualität (vgl. Kosnick 2010) und verknüpft diese mit beispielsweise race/Ethnizität (vgl. Yuval-Davis/Anthias 1989). Die Verteilung von (in)formellen Rechten, also citizenship, spielt eine Rolle dabei, wie soziale Akteur*innen ihr Leben gestalten können. Für die Grenz- und Migrationsforschung bedeutet dies, dass das transnationale Praktizieren von Sexualität (vgl. Barglowski et al. 2018) auch mit Bezug auf die Regulierung von Zugehörigkeit und Migration (vgl. Kosnick 2016) untersucht wird. Beispielsweise werden Rechte wie Familiennachzug von einigen Staaten für heteronormative Familien reserviert (vgl. ebd.). Transnationalität interagiert demnach mit Sexualität, wenn Staaten einander nach ihren formellen Regulationen von Sexualität beurteilen und z.B. Personen aufgrund von Homosexualität Asyl (nicht) gewähren. Weiterhin stellten Karolina Barglowski, Anna Amelina und Başak Bilecen fest, dass transnational lebende Individuen ihre Sexualität nach Kontexten unterschiedlich ausleben (vgl. Barglowski et al. 2018), was Fragen über die Gestaltung von Möglichkeitsräumen und Kämpfen in grenzüberschreitenden, intimen Settings aufwirft. Arnaldo Cruz-Malavé und Martin F. Manalansan (2002) und andere diskutieren diese Themen unter dem Begriff Queer Diaspora.

Diese unterschiedlichen Beispiele veranschaulichen, inwiefern die Interferenz von Geschlechter- und nationalstaatlichen Ordnungen Konsequenzen für transnationale Akteur*innen hat. Grenz(ziehungs)- und Transnationalitätsforschung können einander bei der Beantwortung 
dieser Fragen unterstützen, da Migrant*innen in multiplen Ordnungskontexten gleichzeitig agieren und deshalb komplexe Kämpfe um Zugehörigkeit und Ressourcen navigieren.

\subsection{Transnationalität und race/Ethnizität}

Wie unter Abschnitt 2 bereits angeklungen, interagiert Transnationalität mit der Ungleichheitsachse race/Ethnizität, die u.a. auf koloniale (Ausbeutungs-)Praxis und Wissensordnungen (vgl. Said 1987; Spivak 1988) verweist. Die Institutionalisierung der Ungleichheitsachse race, häufig als Kultur oder Ethnizität bezeichnet (vgl. Balibar 1991), aber keineswegs damit identisch, war eine wichtige Komponente zur Legitimation der Machtasymmetrie zwischen dem Westen und dem Rest der Welt (vgl. Hall 1992). Sie zeigt sich bis heute - neben individuellen, institutionellen und systemischen Formen von Rassismus - in der unreflektierten Handhabung (post)kolonialer Archive und Geschichtsschreibung (vgl. Stoler 2002) oder in der diskursiven Ausblendung postkolonialer Beziehungen aus gegenwärtiger Migration (vgl. Erel et al. 2016). Gurminder Bhambra beispielsweise kritisiert in der aktuellen Debatte um race, dass die Forschung das Erleben und Verhalten von Weißen (in den USA und Großbritannien) als universell zentriert (methodological whiteness; vgl. Bhambra 2016; 2017), während sie die Erfahrungen von rassifizierten und ethnisierten Bevölkerungsteilen ausblendet und die strukturelle Marginalisierung damit reproduziert. Im Gegenzug dazu werden weiße Migrant*innen nicht als Migrant*innen, sondern als mobile Kosmopolit*innen (vgl. Lundström 2017) oder unsichtbare Rückkehrer*innen (vgl. Smith 2003) betrachtet und politisch teilweise privilegiert. Die Interferenz der Ungleichheitsachsen race und Transnationalität führt dabei nicht dazu, dass sie ineinander aufgehen. Sie sind jedoch historisch stark verknüpft, weil epistemologisch Mobilität, Sesshaftigkeit und askriptive Merkmale wie Hautfarbe verschmelzen. Die Kritik an dieser theoretischen und empirischen Lücke verweist auf das allgemeine Problem der Soziologie, die das Soziale von einem Standpunkt aus denkt, der zwischen Traditionalem und Modernem in zeitlicher als auch räumlicher Art hierarchisierend unterscheidet (vgl. Bhambra 2016). Southern Methodologies weisen hier die Richtung, wenn sie darauf reagierend beispielsweise partizipative und dekoloniale Methoden und Epistemologien nutzen (vgl. Mato 2000; Meckesheimer 2013), die die übliche Machtimbalance zwischen Forscher*innen und den an der Forschung Partizipierenden aushebeln.

Transnationalität kann also als Ungleichheitsachse innerhalb der Zuweisung von Lebenschancen betrachtet werden. Sie ist eine Achse, eine Grenzziehung in der Sprache der Grenzforschung, entlang derer sozialräumliche Nähe, (räumliche und temporale) Anwesenheit und Netzwerkzugehörigkeit relevant gemacht werden, um Individuen und Gruppen in der jeweiligen Ordnung zu positionieren. Sesshaftigkeit wird dabei als das statistisch als auch normativ Normale gesetzt und institutionell abgesichert, während Mobilität und Multilokalität erschwert werden.

\section{Leerstellen und Potenziale in der Transnationalitäts- und Migrationsforschung}

Die Transnationalitätsforschung hat den Anspruch, sich von den strukturellen und semantischen Bedingungen des Nationalstaats zu lösen und diese kritisch zu beleuchten. Diese Kritik führt zu fruchtbaren Konzepten wie die der transnationalen Felder oder Sozialräume, die 
sichtbar machen, dass und wie (Im-)Mobile ihr Leben im Kontext transnationaler Aushandlungen gestalten. Mit Theorien mittlerer Reichweite beleuchtet die Transnationalitätsforschung damit einen Teil der Lebensrealität, der durch die traditionelle Migrationsforschung übersehen wurde (vgl. Portes et al. 2017). Die Ausdifferenzierung des Gegenstands, die Entwicklung neuer Methoden und die Reflexion des Verhältnisses vom Nationalstaatscontainer zur sozialen Praxis der Mobilität haben zur fruchtbaren Vermehrung der Fragen beigetragen, während die Erkenntnisse politisch weitgehend ungehört geblieben sind.

Die Grenze und der Nationalstaat an sich bleiben in dieser Theorietradition in der Regel eher eine Blackbox, was eine große Leerstelle in der Transnationalitätsforschung darstellt. Doch obwohl Nationalstaaten im Rahmen von globalem Kapitalismus den Zugriff auf sozioökonomische Phänomene verlieren, ist beispielsweise die zunehmende securitization der Migration in Europa (vgl. Genova 2017) ein Beweis dafür, dass Staaten und suprastaatliche Organisationen weiterhin für die Untersuchung relevant sind (vgl. Dahinden 2017). Transnationalitätsforschung zeigt beispielsweise auch, dass soziale Ungleichheit nicht nur innerhalb von Containerstaaten verhandelt wird (vgl. Weiß 2005). Man kann also von einer reflexiven Vielschichtigkeit in der weltweiten sozialen Praxis sprechen, was sich z.B. anhand von Kämpfen um Klassenstrukturen, Rassismen und heteronormative Ordnungen jenseits nationaler Kontexte darstellen lässt. Die Debatte um Sozialräumlichkeit, die von der Einsicht getragen wurde, dass mobile Menschen nicht alle Brücken hinter sich abbrechen, zeigt deutlich, dass sich durch globalen Kapitalismus und Kommunikationstechnologien die Lebensumstände von Menschen vervielfältigt haben. Die gegenwärtige Transnationalitäts-, Migrations- und Grenzforschung verbindet, dass sie von dieser Komplexitätsdiagnose geleitet werden. Das Zusammenwirken von Institutionen und Praxis kann nur mit einer denationalisierten Epistemologie erforscht werden. Das gelingt dann, wenn Theorien in der Lage sind, scheinbar abweichende Praxis, darunter Mobilität und grenzüberschreitende Beziehungen, zu normalisieren und analytisch zu integrieren.

Die neueste Theoriebildung und Forschung beschäftigt sich expliziter mit den Auswirkungen von institutionellen Regimen auf Transnationalität. Diese sollen zum Schluss diskutiert werden.

\subsection{Transnationalität und ihre Beziehung zur Migrations- und Grenzregimeforschung}

Die Begriffe Migrations- und Grenzregime bezeichnen das „umkämpfte Geflecht aus Akteuren, Praktiken, Diskursen, Materialitäten, Bewegungen und Kämpfen [...,] in und zwischen denen um Kontrolle und Bewegungsfreiheit gerungen wird“ (Hess et al. 2015, S. 2; vgl. Pott et al. 2018). Die Migrations- und Grenzregimeforschung untersucht damit die institutionellen Routinen und Kämpfe, die die Durchlässigkeit von Grenzen bestimmen. Zu den Analyseeinheiten zählen darüber hinaus beispielsweise Wissenschaftler*innen (vgl. Nieswand/Drotbohm 2014) und Migrant*innen (vgl. Tsianos 2010). Während die Transnationalitätsforschung untersucht, wie Individuen und Netzwerke über geografische Distanz operieren, liefert die Migrations- und Grenzregimeforschung wichtige Hinweise darauf, wie die rahmende Praxis von staatlichen und semistaatlichen Akteur*innen organisiert ist. Die Migrations- und Grenzregimeforschung öffnet den Blick auf die fragmentierten Machtbeziehungen und -kämpfe, die Mobilität begleiten und formen (border struggles, vgl. Mezzadra/Neilson 2013). 


\subsection{Doing Migration}

Eine weitere theoretisch und methodologisch hilfreiche Perspektive ist der Doing MigrationAnsatz (vgl. Amelina et al. 2016; Lutz/Amelina 2017; vgl. auch „doing borders“: Heinze et al. 2016; Wonders/Jones 2018). Anna Amelina nutzt die sozialkonstruktivistisch und wissenssoziologisch informierte Einsicht, dass Migration als Beschreibungs- und Bewertungskategorie neben anderen existiert (z.B. Reise, Mobilität, Sesshaftigkeit, Transit). Das Label Migration ist dabei in der Regel negativ besetzt. Sie geht davon aus, dass multiple Ebenen von institutionellem, kollektivem, individuellem und organisationalem Handeln (z.B. lokal bis global) zusammenspielen, um intersektionell verwickelte Mobilitätsordnungen zu erzeugen. Doing Migration verknüpft den Motility-Ansatz von Michael Flamm und Vincent Kaufmann (2006), den mobility turn in den Sozialwissenschaften (vgl. Urry 2007) und Neil Brenners Skalentheorie (1998). Die Analyse verbindet so vier Komponenten: institutionelle, teils territorial-materielle Routinen (z.B. Pässe, Grenzkontrollpraktiken, Zäune), routinisierte individuelle und kollektive Verhaltensweisen (z.B. Identifikation mit Nationen, Landessprachen, Reisen als kulturelle Aneignungspraxis, transnationale Familien), Machtbeziehungen und Klassifikationsordnungen (z.B. die Verteilung von Lebenschancen entlang der Geburtszugehörigkeit, multiskalares Zusammenwirken von politischen Organisationsformen, feldspezifische Machtspiele) und Wissensbestände (z.B. Diskurse über Zugehörigkeit, Containerstaaten, Homogenität und Territorialität, Klassifikationsordnungen). Die politisch-territoriale Grenze wird in diesem Kontext als Citizenship-Praxis thematisiert und gleichzeitig zu anderen Konfigurationen wie Geschlecht ins Verhältnis gesetzt. Methodologisch als auch theoretisch liefert Amelina hier Anknüpfungspunkte für die Grenz- und Mobilitätsforschung, da sie die komplexen Zusammenhänge von Wissen und Macht in der Klassifizierungspraxis und in den dazugehörigen Praktiken bestimmbar macht. Doing Transnationality ist damit eine Praxis des Erzeugens von grenzüberschreitender Sozialräumlichkeit im Spannungsverhältnis zu Migrations- und Grenzregimen.

\subsection{Transnationalität und Digitalisierung}

Nach Faist et al. (2014, S. 19) sind in vielen Fällen nicht Migrant*innen die Hauptakteur*innen transnationaler Praxis, sondern Organisationen (zu Migrant*innenorganisationen vgl. Portes/Fernandez-Kelly 2015). Das Internet unterstützt transnationale Organisationen und Bewegungen wie Diasporas, transnationale kriminelle oder Themennetzwerke beim Austausch von Informationen, Dienstleistungen und Waren. Es startete mit der Hoffnung unter Modernisierungstheoretiker*innen, dass es eine transnationale demokratische Gemeinschaft fördern würde. Stattdessen wurde das Internet beispielsweise zu einem Schmelztiegel für nationalistische und rassistische Bewegungen (vgl. Daniels 2013). Darüber hinaus sind die direkten Onlineaktivitäten zwischen Produzent*innen und Konsument*innen interessant (vgl. Hatzopoulos/Kambouri 2013, Stichwort Digitaler Kapitalismus), die auf absehbare Zeit neue Transaktionsströme und eine andere Grenz- und Zollpolitik schaffen werden. Die Tests von Smart Borders in der EU zeigen, dass auch digitale biometrische Dokumente und Datensätze die Verfahren an Grenzübergängen verändern. Diese Neuerungen an der Schnittstelle von Digitalisierung und Transnationalität rufen dazu auf, die Konsequenzen von Digitalisierung auf 
Privatsphäre, Achsen der Ungleichheit, Grenzen und Bewegungsfreiheit zu untersuchen (vgl. Kloppenburg/Ploeg 2020).

\section{Fazit}

Ob im Rahmen sozialer, institutioneller oder physischer Grenz(ziehung)en: Transnationalität ist eine immer wichtigere Dimension bei der Untersuchung von migrantischem und immobilem Leben. Die Forschung zu Sozialräumlichkeit zeigt, dass der Nationalstaat als Analyseeinheit in seiner Vielschichtigkeit untersucht werden muss. Wie die Grenzregimeforschung veranschaulicht, sind Nationalstaaten bzw. supranationale Grenzregime wie die EU nicht monolithische Gebilde, sondern umkämpfte Geflechte. Transnationale Migration und Mobilität existieren in einem ständigen Spannungsverhältnis dazu. Transnationalität, also der Grad an potenzieller sozialräumlicher Mobilität und grenzüberschreitender Praxis, ist eine Form von Kampf um Möglichkeitsräume, die darüber hinaus mit anderen Achsen der Ungleichheit wie Geschlecht, Sexualität, Klasse und race/Ethnizität interferiert. Die Grenz- und Migrationsregimeforschung, der Doing Migration-Ansatz und Digitalisierung sind Beispiele für Forschungsperspektiven, die die Komplexität von Grenz(ziehungs)prozessen in den Blick nehmen (werden). Border Studies profitieren von der transnationalen Perspektive, da sie mit dem methodologischen Nationalismus bricht und stattdessen die tägliche Praxis in den Kämpfen um Zugang zu Rechten und Ressourcen betrachtet. (Im-)Mobile navigieren ständig territorial-politische Grenzen und soziale Grenzziehungen. Diese mögen im Falle von Migrant"innen besonders ins Gewicht fallen, jedoch sind Immobile ebenso von Grenz(ziehung)en betroffen. Dabei ist Transnationalität ebenso eine Achse der Ungleichheit bzw. Grenzziehung, die ungleiche Lebenschancen erzeugt.

Im Rahmen der Kämpfe um den Begriff Integration in Deutschland formulierte u.a. Naika Foroutan (2016) die Frage, ob Migrationshintergrund als Grenzziehung zwischen Zugehörigkeit und Exklusion überholt sei und Zugehörigkeit vielmehr auch jenseits von Nationalstaaten formuliert werden müsse. Die Einsicht der Grenzforschung, dass Grenzen das Ergebnis von kontinuierlicher Grenzarbeit sind (vgl. Bossong et al. 2017; Gerst et al. 2018), könnte hier fruchtbar sein. Zum einen kann der gewählte Begriff postmigrantisch selbst als Teil der Grenzarbeit rund um die Grenzziehung Nicht-Deutsch/Deutsch betrachtet werden, zum anderen fokussiert er die tägliche (transnationale) Grenz- und Brückenarbeit von Bürger*innen. Die Transnationalitätsforschung verlässt darüber hinaus den nationalstaatlichen Rahmen und beleuchtet vielschichtig, wie Leben jenseits von Grenzen tagtäglich stattfindet - immer mit Bezug zu den Ressourcen, die durch Zugehörigkeit ermöglicht und verwehrt werden. Und da Staaten vermehrt sozialpolitisch kooperieren und Individuen beruflich und aus persönlichen Gründen mobil bleiben, stellt sich die soziale Frage (vgl. Faist 2019) bzw. die Frage nach globalen Formen von Citizenship (vgl. Levitt et al. 2017) längst auch auf anderen Ebenen als der nationalstaatlichen.

\section{Weiterführende Literatur}

Erel, Umut/Murji, Karim/Nahaboo, Zaki (2016): Understanding the Contemporary Race-Migration Nexus. In: Ethnic and Racial Studies 39, H. 8, S. 1339-1360.

Faist, Thomas/Fauser, Margit/Reisenauer, Eveline (2014): Das Transnationale in der Migration. Eine Einführung. Weinheim: Beltz Juventa.

Faist, Thomas (2019): The Transnationalized Social Question. Migration and the Politics of Social Inequalities in the Twenty-First Century. Oxford: Oxford University Press.

Levitt, Peggy/Viterna, Jocely/Mueller, Armin//Lloyd, Charlotte (2017): Transnational Social Protection: Setting the Agenda. In: Oxford Development Studies 45, H. 1, S. $2-19$. 
Lutz, Helma/Amelina, Anna (2017): Gender, Migration, Transnationalisierung. Eine intersektionelle Einführung. Bielefeld: transcript.

\section{Literaturverzeichnis}

Amelina, Anna/Faist, Thomas (2012): De-naturalizing the National in Research Methodologies: Key Concepts of Transnational Studies in Migration. In: Ethnic and Racial Studies 35, H. 10, S. 1707-1724.

Amelina, Anna/Horvath, Ken/Meeus, Bruno (Hrsg.) (2016): An Anthology of Migration and Social Transformation: European Perspectives. Dordrecht u.a.: Springer.

Baldassar, Loretta/Merla, Laura (Hrsg.) (2014): Transnational Families, Migration and the Circulation of Care: Understanding Mobility and Absence in Family Life. Abingdon: Routledge.

Balibar, Etienne (1991): Is there a Neo-racism? In: Balibar, Etienne, Wallerstein, Immanuel (Hrsg.) Race, Nation, Class: Ambiguous Identities. London: Verso, S. 17-28.

Barglowski, Karolina/Amelina, Anna/Bilecen, Başak (2018): Coming Out within Transnational Families: Intimate Confessions under Western Eyes. In: Social Identities 24, H. 6, S. 836-851.

Barglowski, Karolina/ Krzyżowski, Łukasz/Świątek, Paulina (2015): Caregiving in Polish-German Transnational Social Space: Circulating Narratives and Intersecting Heterogeneities. In: Population, Space and Place 21, H. 3, S. 257-269.

Basch, Linda/Glick Schiller, Nina/Szanton-Blanc, Cristina (1994): Nations Unbound: Transnational Projects, Postcolonial Predicaments and Deterrritorialized Nation States. Amsterdam: Gordon and Breach.

Beck, Uwe/Sznaider, Nathan (2006): Unpacking Cosmopolitanism for the Social Sciences: A Research Agenda. In: British Journal of Sociology 57, H. 1, S. 1-23.

Bhambra, Gurminder K. (2016): Postcolonial Reflections on Sociology. In: Sociology 50, H. 5, S. $960-966$.

Bhambra, Gurminder K. (2017): Brexit, Trump, and „Methodological Whiteness“: on the Misrecognition of Race and Class. In: The British Journal of Sociology 68, H. S1, S. 214-232.

Boccagni, Paolo (2012): Even a Transnational Social Field Must Have its Boundaries: Methodological Options, Potentials and Dilemmas for Researching Transnationalism. In: Vargas-Silva, Carlos (Hrsg.): Handbook of Research Methods in Migration. Cheltenham, UK: Edward Elgar, S. 295-318.

Bossong, Raphael/Gerst, Dominik/Kerber, Imke/Klessmann, Maria/Krämer, Hannes/Ulrich, Peter (2017): Complex Borders: Analytical Problems and Heuristics. In: Opiłowska, Elizabieta/Kurcz, Zbigniew/Roose, Jochen (Hrsg.): Advances in European Borderlands Studies. Baden-Baden: Nomos, S. 65-84.

Bourdieu, Pierre (1996): Die Logik der Felder. In: Bourdieu, Pierre und Wacquant, Loic (Hrsg.): Reflexive Anthropologie. Frankfurt/M.: Suhrkamp, S. 124-146.

Brenner, Neil (1998): Between Fixity and Motion: Accumulation, Territorial Organization and the Historical Geography of Spatial Scales. In: Environment and Planning D: Society and Space 16, H. 4, S. 459481.

Bryceson, Debora F./Vuorela, Ulla (Hrsg.) (2002): The Transnational Family: New European Frontiers and Global Networks. Oxford: Berg.

Büscher, Monika/Urry, John (2009): Mobile Methods and the Empirical. In: European Journal of Social Theory 12, H. 1, S. 99-116.

Carroll, William (2014): Whither the Transnational Capitalist Class? In: Socialist Register 50, S. $162-88$.

Castles, Stephen/Haas, Hein de/Miller, Marc J. (2014): The Age of Migration: International Population Movements in the Modern World. Houndmills: Palgrave Macmillan.

Chakrabarty, Dipesh (2000): Provincializing Europe: Postcolonial Thought and Historical Difference. Princeton: Princeton University Press.

Collins, Patricia H. (1990): Black Feminist Thought: Knowledge, Consciousness and the Politics of Empowerment. Boston: Unwin Hyman.

Crenshaw, Kimberle W. (1989): Demarginalizing the Intersection of Race and Sex: A Black Feminist Critique of Antidiscrimination Doctrine, Feminist Theory and Antiracist Politics. In: University of Chicago Legal Forum 1989, H. 1, Artikel 8, S. 139-167.

Cruz-Malavé, Arnaldo/Manalansan, Martin F. (2002): Queer Globalizations: Citizenship and the Afterlife of Colonialism. New York: NYU Press.

Dahinden, Janine (2017): Transnationalism Reloaded: the Historical Trajectory of a Concept. In: Ethnic and Racial Studies 40, H. 9, S. 1474-1485.

Daniels, Janine (2013): Race and Racism in Internet Studies: A Review and Critique. In: New Media \& Society 15, H. 5, S. 695-719.

Eisenstadt, Shmuel N. (2000): Multiple Modernities. In: Daedalus 129, H. 1, S. 1-29.

Erel, Umut/Murji, Karim/Nahaboo, Zaki (2016): Understanding the Contemporary Race-Migration Nexus. In: Ethnic and Racial Studies 39, H.8, S. 1339-1360. 
Faist, Thomas (2000): The Volume and Dynamics of International Migration and Transnational Spaces. Oxford: Clarendon Press.

Faist, Thomas (2019): The Transnationalized Social Question. Migration and the Politics of Social Inequalities in the Twenty-First Century. Oxford: Oxford University Press.

Faist, Thomas/Fauser, Margit/Reisenauer, Eveline (2014): Das Transnationale in der Migration. Eine Einführung. Weinheim: Beltz Juventa.

Flamm, Michael und Kaufmann, Vincent (2006): Operationalising the Concept of Motility: A Qualitative Study. In: Mobilities 1, H. 2, S. 167-189.

Foroutan, Naika (2016): Postmigrantische Gesellschaften. In: Brinkmann, Heinz. U./ Sauer, Martina (Hrsg.): Einwanderungsgesellschaft Deutschland. Entwicklung und Stand der Integration. Wiesbaden: Springer VS, S. 227-254.

Genova, Nicholas de (2017): Introduction: The Borders of „Europe“ and the European Question. In: Ders. (Hrsg.): The Borders of „Europe“: Autonomy of Migration, Tactics of Bordering. Durham, NC: Duke University Press, S. 1-35.

Gerst, Dominik/Klessmann, Maria/Krämer, Hannes/Sienknecht, Mitja/Ulrich, Peter (2018): Komplexe Grenzen. Perspektiven aktueller Grenzforschung. Berliner Debatte Initial 1/18, S. 3-11.

Glick Schiller, Nina/Salazar, Noel B. (2013): Regimes of Mobility Across the Globe. In: Journal of Ethnic and Migration Studies 39, H. 2, S. 183-200.

Haas, Hein de (2010): The Internal Dynamics of Migration Processes: A Theoretical Inquiry. In: Journal of Ethnic and Migration Studies 36, H. 10, S. 1587-1617.

Hall, Stuart (1992): The West and the Rest: Discourse and Power. In: Hall, Stuart/Gieben, Bram (Hrsg.): Formations of Modernity. Cambridge: Polity Press, S. 275-332.

Haselsberger, Beatrix (2014): Decoding Borders. Appreciating Border Impacts on Space and People. In: Planning Theory \& Practice 15, H. 4, S. 505-526.

Hatzopoulos, Pavlos/Kambouri, Nelli (2013): MIG@NET, Transnational Digital Networks, Migration and Gender. Transnational Digital Networks, Migration and Gender Deliverable D13: "Final Synthesis Report”. www.mignetproject.eu/wp-content/uploads/2013/07/MIGNET_D13.pdf, 16.12.2019.

Hess, Sabine/Kasparek, Bernd/Schwertl, Maria/Sontowski, Simon (2015): Europäisches Grenzregime. Einleitung zur ersten Ausgabe. In: movements. Journal for Critical Migration and Border Regime Studies 1, H. 1, S. 1-6.

movements-journal.org/issues/01.grenzregime/02.einleitung.html, 16.12.2019.

Heinze, Tobias/Illigens, Sebastian/Pollok, Michael (2016): Doing Frontiers: On the Performativity of the European Border and Migration Regime. DNGPS Working Paper, S. 1-16.

ILO (2015). ILO Migrant Domestic Workers Across the World: global and regional estimates. Geneva: International Labour Organization. www.ilo.org/wcmsp5/groups/public/---ed_protect/---protrav/---migr ant/documents/briefingnote/wcms_490162.pdf, 16.12.2019.

Kelly, Philip (2012): Migration, Transnationalism, and the Spaces of Class Identity. In: Philippine Studies: Historical \& Ethnographic Viewpoints 60, H. 2, S. 153-185.

Kloppenburg, Sanneke/Ploeg, Irma van der (2020): Securing Identities: Biometric Technologies and the Enactment of Human Bodily Differences. Science as Culture 29, H. 1 S. 57-76.

Kosnick, Kira (2010): Sexuality and Migration Studies. The Invisible, the Oxymoronic and Heteronormative Othering. In: Lutz, Helma/Herrera Vivar, Maria T./Supik, Linda (Hrsg.): Framing Intersectionality. Debates on a Multi-faceted Concept in Gender Studies. Farnham: Ashgate, S. 121-135.

Kosnick, Kira (2016): Sexual Citizenship and Migration in a Transnational Perspective. Working Paper Series „Gender, Diversity and Migration“ Nr. 9. www.fb03.uni-frankfurt.de/60214700/Working_Paper -No_9.pdf, 16.12.2019.

Levitt, Peggy/Glick Schiller, Nina (2004): Conceptualizing Simultaneity: A Transnational Social Field Perspective on Society. In: International Migration Review 38, H. 3, S. 1002-1039.

Levitt, Peggy/Viterna, Jocely/Mueller, Armin/Lloyd, Charlotte (2017): Transnational Social Protection: Setting the Agenda. In: Oxford Development Studies 45, H. 1, S. 2-19.

Lundström, Catrin (2017): The White Side of Migration: Reflections on Race, Citizenship and Belonging in Sweden. In: Nordic Journal of Migration Research 7, H. 2, S. 79-87.

Lutz, Helma (2018): Masculinity, Care and Stay-Behind Fathers: A Postsocialist Perspective. Critical Sociology 44, H. 7-8, S. 1061-1076.

Lutz, Helma/Amelina, Anna (2017): Gender, Migration, Transnationalisierung. Eine intersektionelle Einführung. Bielefeld: transcript.

Lutz, Helma/Palenga-Möllenbeck, Ewa (2010): Care Work Migration in Germany: Semi-Compliance and Complicity. In: Social Policy \& Society 9, H. 3, S. 419-430.

Lutz, Helma/Herrera Vivar, Maria T./Supik, Linda (2011): Framing Intersectionality: Debates on a MultiFaceted Concept in Gender Studies. Farnham: Ashgate. 
Madianou, Mirca/Miller, Daniel (2011): Migration and the New Media: Transnational Families and Polymedia. Abingdon: Routledge.

Marcus, George E. (1995): Ethnography in/of the World System: the Emergence of Multi-Sited Ethnography. In: Annual Review of Anthropology 24, S. 95-117.

Mato, Daniel (2000): Not "Studying the Subaltern", but studying with "Subaltern" Social Groups, or, at Least, Studying the Hegemonic Articulations of Power. In: Nepantla: Views from South 1, H. 3, S. 479-503.

Meckesheimer, Anika (2013): Decolonization of Social Research Practice in Latin America: What can we Learn for German Social Sciences? In: Transcience 4, H. 2. www2.hu-berlin.de/transcience/Vol4_Issue2 2013_79_98.pdf, 16.12.2019.

Mezzadra, Sandro/Neilson, Brett (2013): Border as Method, or, the Multiplication of Labor. Durham und London: Duke University Press.

Munck, Ronaldo (2013). The Precariat: a View from the South. In: Third World Quarterly 34 H. 5, S. 747-762.

Nieswand, Boris/Drotbohm, Heike (2014): Kultur, Gesellschaft, Migration. Die reflexive Wende in der Migrationsforschung. Wiesbaden: Springer VS.

Oguntoye, Katharina/Ayim/ May/Schultz, Dagmar (1986): Farbe bekennen. Afro-deutsche Frauen auf den Spuren ihrer Geschichte. Berlin: Orlanda.

Olwig, Karen F. (2014): Migration and Care: Intimately Related Aspects of Caribbean Family and Kinship. In: Baldassar, Loretta/Merla, Laura (Hrsg.): Transnational Families, Migration and the Circulation of Care: Understanding Mobility and Absence in Family Life. Abingdon: Routledge, S. 133-148.

Parreñas, Rhacel S. (2005): Children of Global Migration: Transnational Families and Gendered Woes. Stanford: Stanford University Press.

Portes, Alejandro/Fernández-Kelly, Patricia (2015): The State and the Grassroots: Immigrant Transnational Organizations in Four Continents. New York: Berghahn Books.

Portes, Alejandro/Guarnizo Luis E./Landolt, Patricia (2017): Commentary on the Study of Transnationalism: Pitfalls and Promise of an Emergent Research Field. In: Ethnic and Racial Studies 40, H. 9, S. 1486-1491.

Pott, Andreas/Rass, Christoph/Wolff, Frank (2018): Was ist ein Migrationsregime? What is a Migration Regime? Wiesbaden: Springer VS.

Pries, Ludger (2008): Die Transnationalisierung der sozialen Welt: Sozialräume jenseits von Nationalgesellschaften. Frankfurt/M.: Suhrkamp.

Randeria, Shalini (2002): Entangled Histories of Uneven Modernities: Civil Society, Caste Solidarities and Legal Pluralism in Post-Colonial India. Konferenzbeitrag, Civil Society Network Conference, Wissenschaftszentrum für Sozialforschung Berlin. www.urbanlab.org/articles/Randeria ,\%20S.\%202002 \%20EntangledHistories.pdf, 16.12.2019.

Resch, Christine (2014) Reflexivität als Denkmodell und Perspektive in den Sozialwissenschaften. In: Widersprüche: Zeitschrift für sozialistische Politik im Bildungs-, Gesundheits- und Sozialbereich 34, H.132, S. 75-89.

Robinson, William I. (2004): A Theory of Global Capitalism. Baltimore: Johns Hopkins University Press.

Said, Edward W. (1978): Orientalism. New York: Pantheon Books.

Sassen, Saskia (2001): The Global City. New York, London, Tokyo. Princeton: Princeton University Press.

Shinozaki, Kyoko (2015): Migrant Citizenship from Below: Family, Domestic Work, and Social Activism in Irregular Migration. New York: Palgrave Macmillan.

Sklair, Leslie (2001): The Transnational Capitalist Class. Oxford: Blackwell.

Smith, Andrea L. (2003): Europe's Invisible Migrants. Amsterdam: Amsterdam University Press.

Spivak, Gayatri C. (1988): Can the Subaltern Speak? In: Nelson, Cary/Grossberg, Lawrence (Hrsg.): Marxism and the Interpretation of Culture. Chicago: University of Illinois Press, S. 271-316.

Standing, Guy (2011): The Precariat: The New Dangerous Class. New York: Bloomsbury.

Stoler, Ann L. (2000): Colonial Archives and the Arts of Governance. In: Archival Science 2, S. 87-109.

Triandafyllidou, Anna/Marchetti, Sabrina (2015): Employers, Agencies and Immigration: Paying for Care. Farnham: Ashgate.

Tsianos, Vassilis (2010): Zur Genealogie und Praxis des Migrationsregimes. In: Bildpunkt 2010, H. 1, S. 22-24.

Urry, John (2007): Mobilities. Cambridge und Malden, MA: Polity Press.

Weiß, Anja (2005): The Transnationalization of Social Inequality: Conceptualizing Social Positions on a World Scale. In: Current Sociology 53, H. 3, S. 707-728.

Wimmer, Andreas und Glick Schiller, Nina (2003): Methodological nationalism, the social sciences, and the study of migration: An essay in historical epistemology. In: International Migration Review $37, \mathrm{H}$. 3, S. 576-610. DOI: 10.1111/j.1747 - 7379.2003.tb00151.x. 
Wonders, Nancy A. und Jones, Lynn C. (2018): Doing and Undoing Borders: The Multiplication of Citizenship, Citizenship Performances, and Migration as Social Movement. In: Theoretical Criminology 23, H. 2, S. 136-155. DOI: 10.1177/1362480618802297.

Yuval-Davis, Nira/Anthias, Floya (1989): Woman-Nation-State. Houndsmills u.a.: Palgrave Macmillan. 